\title{
Apuntes para la enseñanza de objetos matemáticos inmersos en el álgebra escolar. Un paso por diversas investigaciones ${ }^{1}$
}

\author{
Notes for teaching mathematical objects immersed in \\ school algebra. A step by various research
}

Notas para o ensino de objetos matemáticos imersos em álgebra escolar. Um passo por várias pesquisas

Recibido: mayo de 2013

Aceptado: agosto de 2013
Paula Andrea Aponte Bello ${ }^{2}$

Hasbleidy Lizeth Alméciga Ruiz ${ }^{3}$

Deisy Torres Rodríguez ${ }^{4}$

\section{Resumen}

Esta comunicación versa sobre diversas investigaciones: Kieran, Filloy, Baursfeld y Skowrone k, Wheeler y Socas, entre otros, que dan cuenta de obstáculos, errores comunes, entendimientos, concepciones y estrategias de trabajo, en torno a procesos de enseñanza-aprendizaje del álgebra escolar. A partir del estudio histórico-epistemológico del álgebra, como recurso didáctico, se presenta una propuesta de enseñanza la cual permitirá ser implantada al inicio de la enseñanza del algebra en estudiantes de secundaria, contemplando lo curricular como conjunto de experiencias, la transposición y las organizaciones didácticas como necesidades, del y para el aula. Así el fin último de este escrito, es develar elementos mínimos requeridos al momento de enseñar álgebra escolar teniendo como referente didáctico principal el uso de la historia y los obstáculos que allí se presentan, elementos estudiados y configurados en el curso Didáctica del Álgebra.

Palabras clave: Matemáticas escolares; álgebra; recursos didácticos; historia; enseñanza; metodología de enseñanza; metodología de trabajo en el aula.

\begin{abstract} Skowrone Baursfeld and $\mathrm{k}$, Wheeler and Socas, among others, that account for obstacles, common mistakes, understandings, concepts and strategies work around teaching-learning algebra school. From the historical-epistemological study of algebra, as a teaching resource, it presents a teaching proposal which will be implemented at the beginning of the teaching of algebra in high school students, contemplating the curriculum as a set of
\end{abstract}

This communication concerns various investigations: Kieran, Filloy 
experiences, transposition and organizations teaching as needs of and for the classroom. So the ultimate goal of this paper is to reveal the minimum required elements when teaching school algebra having as main teaching regarding the use of history and there are obstacles, and set elements studied in the course Teaching of Algebra.

Keywords: school mathematics; algebra teaching resources, story, teaching, teaching methodology, methodology of work in the classroom

\section{Resumo}

Esta comunicação refere-se várias investigações: Kieran, Filloy Skowrone Baursfeld e k, Wheeler e Socas, entre outros, que representam obstáculos, erros comuns, entendimentos, conceitos e estratégias de trabalho em torno de álgebra de ensino-aprendizagem escola. A partir do estudo histórico-epistemológica da álgebra, como recurso didático, apresenta uma proposta de ensino que será implementado no início do ensino de álgebra em alunos do ensino médio, contemplando o currículo como um conjunto de experiências, transposição e organizações ensinando como as necessidades de e para a sala de aula. Assim, o objetivo final deste trabalho é revelar os elementos mínimos necessários para ensinar álgebra escolar, tendo como principal ensinamento sobre o uso da história e existem obstáculos e definir os elementos estudados no Ensino curso de Álgebra.

Palavras-chave: matemática escolar; recursos de ensino de álgebra, história, ensino, metodologia de ensino, metodologia de trabalho em sala de aula.

\section{Introducción}

Para dar comienzo al desarrollo de este documento, es necesario sumergirse en el proceso enseñanza- aprendizaje que se llevó a cabo en el curso didáctica del álgebra, el cual se desenvolvió a partir de una secuencia de actividades diseñadas para ser resueltas tanto a nivel personal como grupal. En el transcurso de este proceso surgen una cadena de planteamientos de situaciones problemas, para las cuales se requería el uso de conceptos que a nivel universitario deberían ser dominados, pero, al no ser así, salieron a flote dificultades y obstáculos, que provenían directamente de la educación anterior que fue impartida a cada uno de los participantes.

Es allí donde surge el siguiente cuestionamiento, y del cual se partirá para el diseño de una propuesta que mitigue algunas de estas dificultades: ¿Cómo presentar conceptos algebraicos formales a los estudiantes, logrando así una correcta interiorización y posterior aplicación en diferentes contextos? Para hacer un abordaje adecuado de la pregunta orientadora, se remitirá como primera instancia a estudios realizados sobre el mismo tema, posteriormente se tomará el informe realizado por (Bauersfeld y Skowronek 1976) titulado "Investigaciones relacionadas con el proceso de aprendizaje de las matemáticas", este documento permite identificar los pasos por los cuales atraviesa el estudiante en la adquisición de un determinado concepto matemático, además de mencionar algunas de las concepciones inadecuadas que poseen los estudiantes en el aprendizaje del álgebra, donde se encuentra que los estudiantes tienen una precaria apropiación del conocimiento algebraico.

El problema que se aborda, requiere establecer estrategias de enseñanza, es decir diseño de nuevas propuestas metodológicas que contribuyan a desarrollar las competencias del estudiante, de tal manera que su aprendizaje sea significativo. (Llanos Vargas).

De acuerdo con lo antes mencionado se hace énfasis en algunas de las dificultades que se manifestaron con más frecuencia dentro del curso, a partir de una serie de trabajos expuestos en el aula frente 
al estudio inicial del álgebra,donde, el estudiante no logra aplicar esta rama en el interior de las matemáticasmismas ni en otras áreas del saber al traer consigo nociones y enfoques que usaba en aritmética. Como sustento de las afirmaciones anteriores se toma a (Pérez 1997) el cual afirma que el conocimiento Algebraico es esencial por su aporte a la comunicación y expresión de las matemáticas, a la construcción de modelos y a la estructuración de formas de razonamiento, de igual manera KIERAN \& FILLOY YAGUE, establecen que el álgebra no es simplemente una generalización de la aritmética y que aprenderla no implica hacer explícito lo que está contenido en la aritmética, esto podría requerir un cambio en el proceso del estudiante en las situaciones numéricas concretas a proposiciones generales sobre números y operaciones.

Como segunda instancia, se evidencia que el educando presenta dificultad al identificar el signo igual como una equivalencia entre el lado izquierdo y derecho de una ecuación (Kieran 1980). Desde la perspectiva de los estudiantes, estos conciben el signo de igualdad como una herramienta que le permite separar una secuencia de operaciones y el resultado les lleva a romper con las propiedades simétricas y transitivas de la igualdad. La contingencia de este hecho, lleva a que los estudiantes por el deseo de generalizar lo que es correcto en aritmética, malinterpreten el uso de los símbolos en el inicio del álgebra.

Finalmente, se tiene el uso de los paréntesis, y el orden de las operaciones, en donde los estudiantes no consideran que estos son necesarios para denotar el orden en que se efectúan las operaciones (Kieran, 1979)

Es pertinente aclarar, que aunque sólo se mencionan 3 dificultades que manifiestan los estudiantes en la transición de la aritmética al álgebra, no son las únicas presentes en el desarrollo de dicho conocimiento.

Ahora, desde la postura y experiencias personales en el curso anteriormente mencionado, se puede ver que los estudiantes en su afán por dar respuesta a los diferentes problemas planteados por el docente, se ven obligados a emplear diferentes métodos informales, es decir, métodos no enseñados.
Estos métodos permiten que el estudiante se centre en conseguir únicamente respuesta al problema sin la necesidad de especificar los procedimientos que se utilizaron (conocimientos previos) y aquellos que fueron desarrollados (nuevos conocimientos), que en realidad es a lo que se pretende llegar, ya que no solo se busca ver una respuesta correcta o incorrecta, sino también los procedimientos que implementó el alumno para finalmente llegar a su propio razonamiento. Esto ayuda a identificar errores, que han surgido en todo el desarrollo del trabajo expuesto por cada uno de los estudiantes, lo que llevó a reconocer que en el aprendizaje del álgebra, siempre va a ser indispensable formalizar procedimientos por los cuales, tal vez nunca se hayan preocupado. Si esto no es así, entonces no solo tendríamos que dedicarnos a mirar lo que exteriorizan los educandos, sino también, ¿qué es en realidad lo que sucede con el pensamiento algebraico? para lo cual afirma

Hoy en día el álgebra no es meramente "dar significado a los símbolos" sino otro nivel más allá de eso; que tiene que ver con aquellos modos del pensamiento que son esencialmente algebraicos -por ejemplo, manejar lo todavía desconocido, invertir y deshacer operaciones, ver lo general en lo particular. Ser consciente de esos procesos, y controlarlos, es lo que significa pensar algebraicamente. Wheeler, (citado por Kieran 1986)

Es a partir de lo que nos plantean anteriormente, que creemos indispensable que el estudiante consiga ver lo general en lo particular (Wheeler, 1986), de esta manera logrará no sólo adentrarse en lo que significa comprender estructuralmente el álgebra, sino será capaz de darle uso en cualquier situación requerida.

Se es consciente que un aprendizaje estructural del álgebra no se da apresuradamente, pero es a partir de ello, que se cree necesario diseñar una propuesta la cual el docente pueda implantar en el aula, donde se mitiguen en un buen porcentaje estas dificultades.

Esta propuesta está basada en el recorrido que Descartes realizó en la construcción del lenguaje simbólico, ya que anteriormente se habían 
realizado algunas investigaciones en lo que refiere a personajes influyentes en la creación del álgebra; en comparación con otros sujetos este logró sobresalir ya que no solo realizó investigaciones anteriores sino que a partir de los avances de sus antecesores creó un nuevo lenguaje que encerró lo ya creado hasta el momento y los nuevos razonamientos propuestos por él. Dentro de esta investigación se logró observar que dicho proceso atravesado por Descartes podría ser fructífero en la realización de una propuesta, tomando como base los diferentes momentos históricos por los cuales tuvo que atravesar Descartes. Uno de estos es el siguiente artículo en el cual se afirma lo siguiente:

Al-Khwarizmi es el primero en enseñar álgebra de una manera elemental y por sí misma. En los estudiantes sería bueno comenzar su enseñanza de una "manera elemental", al modo de al-Khwarizmi, con los aportes que hasta aquí mencionamos, desarrollando la posibilidad de un trabajo simultáneo en dos marcos, geométrico y algebraico, e incorporando el modelo de área bajo distintas representaciones. (Covas y Bressan, 2002.pg.12)

Descartes logró realizar un lenguaje que le permitió representar de una manera clara y eficaz de lo que estaba hablando, llevándolo a apropiarse de un nuevo lenguaje. Aterrizando el proceso que llevó Descartes a la propuesta que se pretende enunciar en este documento a continuación se darán los aspectos relevantes que se tuvo en cuenta en dicha formulación:

Esta propuesta consiste en enseñar a los estudiantes el álgebra mediante el recorrido que Descartes realizó en la construcción del lenguaje simbólico. Una propuesta que sirve de sustento se encuentra en el documento titulado "La enseñanza del álgebra y los modelos de área"

Llevando el mismo proceso que surtió un efecto positivo en Descartes se rescata la siguiente metodología favorable para realizar con los estudiantes adaptados a unas condiciones que se generan en el aula.

Consiste en iniciar con el estudio del álgebra mediante el uso del lenguaje retórico, generando en el estudiante la necesidad de comprimir el proceso que está realizando y encontrar un lenguaje que le sea propicio para plasmar sus ideas en cuanto a la resolución de un problema determinado.

A continuación se presenta una relación de los diferentes momentos que debe atravesar el docente con sus estudiantes en la enseñanza del álgebra. Iniciando con el lenguaje retórico seguido del sincopado y por último el lenguaje simbólico. Esta idea surge tanto de las vivencias grupales como individuales a lo largo de las sesiones en la materia didáctica del álgebra; pues a medida que cada grupo de trabajo mostraba los avances obtenidos en cada tema correspondiente, se veían reflejadas las dificultades ya mencionadas, de tal manera que las autoras deciden realizar una recopilación de dichas dificultades y a través diferentes investigaciones tanto a dificultades como a propuestas por el mismo eje y contando con la ayuda del docente se realizó esta propuesta.

Es importante que el docente sepa adaptar esta propuesta a las necesidades del aula teniendo en cuenta el tipo de población al que se enfrenta para que de esta manera se generen los resultados esperados.

El éxito de esta propuesta depende en gran medida de la labor docente ya que el profesor debe elegir adecuadamente los temas que quiere presentar mediante esta guía.

Dentro del recorrido realizado en el documento finalmente se llega a las siguientes conclusiones en cuanto a los distintos temas abordados:

- No es pertinente iniciar con los estudiantes en el estudio del álgebra, dando definiciones sin ninguna relación y sentido apreciable.

- Es importante trabajar el álgebra por medio de la resolución de problemas, con situaciones significativas, las cuales sean atractivas para los estudiantes e incentiven.

- No se trata de que el docente brinde a los estudiantes todos los temas del álgebra, por el contrario se trata que el docente realice una elección estratégica que brinde las bases necesarias para 
que el estudiante logre desenvolverse en cualquier tipo de problema que más adelante se le plantee.

\section{Referencias}

Alonso, F. Barrero, C. Fuentes, I. Azacarate, G. Dozagarat, G. Gutierrez, S. Ortiz, M. Riviere, V. Veiga, A. (1993). Ideas y actividades para enseñar álgebra. España: Síntesis.

Covas. M. C., \& Bressan. A. (2002). La enseñanza del álgebra y los modelos de área. Recuperado en http://www.gpdmatematica.org.ar/publicaciones/algebrageometricacovas3.pdf
Malisani, E. (1999). Los obstáculos epistemológicos en el desarrollo del pensamiento algebraico, visión histórica. IRICE $N^{\circ} 13$.

Kieran, C. Filloy, Y. E. (1989). El aprendizaje del álgebra escolar desde una perspectiva psicológica; Investigaciones y experiencias didácticas. Recuperado de http://ddd.uab.es/pub/edlc/02124521v7n3p229.pdf

Socas, M. Camacho, M. Palarea, M. Hernandez, (1996). Iniciación al álgebra. España: Editorial Síntesis. 
Anexo 1

\begin{tabular}{|c|c|c|}
\hline $\begin{array}{c}\text { TIPO DE } \\
\text { LENGUAJE }\end{array}$ & INTENCIÓN & $\begin{array}{c}\text { ASPECTOS A TENER EN } \\
\text { CUENTA }\end{array}$ \\
\hline $\begin{array}{l}\text { Lenguaje } \\
\text { Retórico }\end{array}$ & $\begin{array}{l}\text { Se da como fase de iniciación al } \\
\text { álgebra formal en los estudiantes, el } \\
\text { inicio que tuvo el álgebra, donde se } \\
\text { hará un recorrido, buscando que } \\
\text { aprecien la evolución que se dio en } \\
\text { el álgebra en cuanto a su forma de } \\
\text { representación. } \\
\text { Es importante que el docente realice } \\
\text { una elección estratégica de las } \\
\text { situaciones problema que va a } \\
\text { presentar a los estudiantes teniendo } \\
\text { encuenta los conceptos con lo cuales } \\
\text { desea iniciar. Por ejemplo si el } \\
\text { docente desea iniciar con el } \\
\text { reconocimiento de expresiones } \\
\text { algebraicas debe plantear a los } \\
\text { estudiantes una situación que } \\
\text { permita que el estudiante modele } \\
\text { finalmente una expresión algebraica } \\
\text { que es lo que se pretende. }\end{array}$ & $\begin{array}{l}\text { El docente debe plantear situaciones } \\
\text { donde el estudiante modele } \\
\text { problemas algebraicos pero con } \\
\text { lenguaje retórico, y dé las } \\
\text { soluciones a dichos problemas con } \\
\text { el mismo tipo de lenguaje. } \\
\text { Plantear cuestionamientos a los } \\
\text { estudiantes buscando que se dé la } \\
\text { construcción conjuntamente del } \\
\text { concepto sin olvidar hacer al final de } \\
\text { cada proceso una } \\
\text { institucionalización en donde se } \\
\text { cierren los temas vistos a lo largo del } \\
\text { proceso. }\end{array}$ \\
\hline $\begin{array}{l}\text { Lenguaje } \\
\text { Sincopado }\end{array}$ & $\begin{array}{l}\text { Se pretende que el estudiante busque } \\
\text { la manera de comunicar sus ideas } \\
\text { abandonando el lenguaje retórico, } \\
\text { expresando otro tipo de lenguaje en } \\
\text { el cual enuncia lo mismo, de una } \\
\text { forma práctica comunicando sus } \\
\text { ideas matemáticas. }\end{array}$ & $\begin{array}{l}\text { El docente debe partir de la misma } \\
\text { situación problema planteada para la } \\
\text { fase de iniciación, y de ahí lograr } \\
\text { que los estudiantes modelen los } \\
\text { problemas algebraicos vistos desde } \\
\text { la apertura, pero en lenguaje } \\
\text { sincopado logrando hacer un } \\
\text { paralelo entre los dos lenguajes. } \\
\text { Permitir una socialización, en el que } \\
\text { cada estudiante manifieste sus } \\
\text { cuestionamientos y los caminos } \\
\text { abordados. }\end{array}$ \\
\hline $\begin{array}{l}\text { Lenguaje } \\
\text { Simbólico }\end{array}$ & $\begin{array}{l}\text { Esta fase es muy importante ya que } \\
\text { al estudiante se le presenta el } \\
\text { lenguaje que caracteriza el álgebra y } \\
\text { con el cual desde ese momento } \\
\text { deberá comprender todos los } \\
\text { razonamientos que allí se le } \\
\text { planteen. }\end{array}$ & $\begin{array}{l}\text { Se seguirá trabajando con los } \\
\text { mismos problemas planteados desde } \\
\text { el inicio del curso, donde el } \\
\text { estudiante logre un manejo } \\
\text { adecuado del lenguaje simbólico, } \\
\text { teniendo presente las dificultades } \\
\text { mencionadas a lo largo del proceso. } \\
\text { Aquí se hará un análisis entre el } \\
\text { docente y los estudiantes frente a } \\
\text { los tres tipos de lenguajes. Es } \\
\text { importante que el docente de un } \\
\text { tratamiento especial a este proceso } \\
\text { ya que este le permitirá } \\
\text { desenvolverse en los siguientes } \\
\text { temas algebraicos dando un uso } \\
\text { correcto al lenguaje simbólico. }\end{array}$ \\
\hline
\end{tabular}

